\title{
Management of patients with COVID-19 in radiology departments, and indications regarding imaging studies - recommendations of the Polish Medical Society of Radiology
}

\author{
Andrzej Cieszanowski , Elżbieta Czekajska², Barbara Giżycka³ , Katarzyna Gruszczyńska4 , Joanna Podgórska1, \\ Agnieszka Oronowicz-Jaśkowiak' ${ }^{1}$ Zbigniew Serafin ${ }^{5}$, Edyta Szurowska ${ }^{6}$, Jerzy M. Walecki ${ }^{7}$
}

\author{
1Radiology Department 1, Maria Sklodowska-Curie National Research Institute of Oncology, Warsaw, Poland \\ ${ }^{2}$ Department of Radiology, Medical University of Lublin, Lublin, Poland \\ ${ }^{3}$ Department of Diagnostic Imaging, Multispeciality Hospital Megrez Sp. z 0.0., Tychy, Poland \\ ${ }^{4}$ Department of Radiology and Nuclear Medicine, Faculty of Medical Science in Katowice, Medical University of Silesia, Katowice, Poland \\ ${ }^{5}$ Department of Radiology and Diagnostic Imaging, Nicolaus Copernicus University, Collegium Medicum, Bydgoszcz, Poland \\ ${ }^{6} 2^{\text {nd }}$ Department of Radiology, Medical University of Gdansk, Gdansk, Poland \\ 7Department of Diagnostic Imaging, Centre of Postgraduate Medical Education, Warsaw, Poland
}

\section{Abstract}

\begin{abstract}
The pandemic involving COVID-19 caused by the SARS-CoV-2 coronavirus, due to its severe symptoms and high transmission rate, has gone on to pose a control challenge for healthcare systems all around the world. We present the second version of the Recommendations of the Polish Medical Society of Radiology, presuming that our knowledge on COVID-19 will advance further rapidly, to the extent that further supplementation and modification will prove necessary. These Recommendations involve rules of conduct, procedures, and safety measures that should be introduced in radiology departments, as well as indications for imaging studies.
\end{abstract}

Key words: COVID-19, SARS-CoV-2, PMSR, PLTR.

\section{Introduction}

The pandemic involving the novel coronavirus disease 2019 (COVID-19) had its roots in Wuhan, China, in November 2019, and is attributed to what came to be called severe acute respiratory syndrome coronavirus 2 (SARS-CoV-2) [1]. Early patients presented with symptoms of severe pneumonia, including fever, dry cough, and respiratory distress. Due to the high transmission rate characterising the virus, it has gone on to pose a control challenge for healthcare systems around the world [2]. Although infection rates in China are said to have been controlled by way of stringent public-health measures, several European countries have seen exponential in- creases in numbers of infected and deceased COVID-19 patients recently. It is thus crucial for other countries to prepare their responses to the pandemic, in circumstances in which effectiveness is seen to depend crucially on rapidly-introduced prevention and control measures, including early detection, diagnosis, treatment, and quarantine, as well as a reduction in secondary infections among healthcare professionals [3].

A real-time reverse transcription-polymerase chain reaction (RT-PCR) assay for COVID-19 has been developed and is in use in clinics [4]. Some authors thus underline that, as PCR tests have become the only diagnostic criteria for SARS-CoV-2 infection, imaging should not

Correspondence address:

Dr. Joanna Podgórska, Radiology Department 1, Maria Sklodowska-Curie National Research Institute of Oncology, 5 Roentgena St., 02-781 Warsaw, Poland,

e-mail:.jpodgo@gmail.com

Authors' contribution:

A Study design · B Data collection · C Statistical analysis · D Data interpretation · E Manuscript preparation · F Literature search · G Funds collection 
be a primary means of diagnosis [5], unless the patient is in a critical condition or PCR tests are unavailable. In line with this view, the primary role of both X-ray and computed tomography (CT) imaging studies is to assist in establishing how to manage the patient, the assessment of complications, and differential diagnosis [5]. Other authors $[6,7]$ point out that the sensitivity of PCR tests remains low, in contrast to chest CT, which may be helpful in rectifying false negatives obtained from RT-PCR during the disease's early stages of development.

Knowledge on the COVID-19 disease caused by the SARS-CoV-2 coronavirus, as well as on the management of infected patients, remain new issues. We thus present the second (April $6^{\text {th }} 2020$ ) version of the Recommendations of the Polish Medical Society of Radiology (PMSR), presuming that our knowledge will advance further rapidly, to the extent that further supplementation and modification will prove necessary.

Given that several hospitals in Poland have been assigned dedicated COVID-19 facilities, it will be clear that the specifics of patient management will differ in these, as opposed to those in multi-profile hospitals. Facilities' topography will also need to be considered, along with ways in which this influences workflow. For these reasons, standard procedures will need to be established for each radiology department (RD), and adapted to local conditions. These procedures will nevertheless need to encompass:

1. General rules of conduct minimising the risk of infection to staff.

2. Safety measures surrounding patients with suspected or confirmed SARS-CoV2 infections.

3. The use of personal protective equipment (PPE) by staff.

4. The use of portable X-ray machines.

5. Patient transport to and from radiology departments; and safety measures applying to waiting rooms.

6. Imaging studies on stationary $\mathrm{X}$-ray/CT scanners; with subsequent decontamination of machines.

7. Indications for imaging studies in patients with suspected or confirmed SARS-CoV-2 infection.

\section{General rules for the protection of staff}

- Personnel receive mandatory training in the use of personal protective equipment (PPE) [8] - especially the removal of masks, goggles, face shields, gowns, and aprons.

- Masks, including both surgical masks and particulate respirators designated for contact with infectious respiratory diseases (with FFP2/FFP3 filters compliant with European standards or N95/N99 - their equivalent in the American nomenclature), fit tight to the face to minimise air "leaks" [9].

- Male healthcare professionals should shave off beards and/or moustaches, as saliva droplets may settle on facial hair.
- Because there is a risk of the infection being transmitted via paper records [10], electronic versions are used wherever possible (in referrals, reports, communications between personnel, etc.).

- Hands need to be washed and disinfected frequently.

- The wearing of jewellery, watches, etc. is contraindicated [7].

- Clothing is changed before personnel leave work.

- Surfaces (desk tops, computer keyboards, etc.) are disinfected regularly [9].

- Radiological reporting online should be enabled (especially when isolation is needed) [8].

- Distances between people (preferably of $\geq 2 \mathrm{~m}$ ) are maintained if at all possible [9].

- Patients in whom SARS-CoV2 infection is suspected wear a mask [7].

- During a COVID-19 epidemic, every patient with cough or dyspnoea wears a surgical mask while undergoing an imaging study.

- Imaging studies in COVID-19 patients are best performed in less-attended areas of RDs, to avoid unnecessary exposure of personnel and other patients [8].

- After examinations have taken place, machines are disinfected, prior to decontamination (if available in the hospital), and 30-60 minutes of air exchange [8,9].

- Team briefings and other (e.g. multidisciplinary) meetings are cancelled during an epidemic [8], with information instead transmitted via videoconferencing and e-mail.

- Whenever possible, technicians, nurses, and physicians work in one place, with job-rotation avoided. Staff should be divided into non-communicating teams [11]. Premises (e.g. social rooms) that gather staff working in different areas are probably closed.

- Wherever possible, radiologists working in infectiousdisease hospitals consider isolating from their families while a SARS-CoV-2 epidemic persists.

\section{Safety measures surrounding patients with suspected or confirmed COVID-19 infection}

- The patient wears a surgical mask at all times and treats this as disposable $[8,9]$. Masks are tight-fitting (to minimise lateral "air leaks") and remain in use for a maximum of one hour. They are then disposed of as infectious waste, with a new mask administered to each patient.

- Patients for examination are moved along designated communication routes [7].

- Where numbers of scanners in an RD are sufficient, machines for COVID-19 patients and for others are designated as separate [7].

- A patient infected with SARS-CoV-2 virus awaiting examination does not leave a designated area of the $\mathrm{RD}$ waiting room [8]. No other patients may be in the waiting room at the same time [8]. 


\section{Personal protective equipment (PPE) for staff}

The RD staff in contact with patients with suspected or confirmed COVID-19 infection are:

- radiographers (who position patients for examination);

- physicians in direct contact with patients (e.g. during ultrasound examinations);

- nurses in direct contact with patients (inserting catheters for contrast administration);

- cleaners disinfecting rooms after examinations. Putting on personal protective equipment [13]:

- Hands are disinfected before any PPE is handled.

- The gown goes on first.

- The FFP2/3 respirator and goggles/face shield are put on, then gloves last of all.

- Protective masks (surgical masks or FFP2/FFP3 respirators) fit tightly to minimise "air leakage" [14].

- Goggles fit with the mask and overlap it.

- Gloves cover wrists and gown cuffs.

Direct instructions for the removal of personal protective equipment [13]:

- The removal of PPE requires special attention because infectious material is present on its outside. Infections often occur as PPE is removed [15].

- Place disposable equipment in infectious waste immediately after removal.

- Discard reusable equipment in a container for contaminated medical equipment, to be decontaminated in line with instructions in the manufacturer's manual.

- Disinfect gloved hands before the outer pair of gloves are removed, ensuring minimised contact between the contaminated side and the inner gloves.

- Disinfect hands again.

- Remove the gown by pulling from its back part and peeling the gown away from the body, at the same time turning the gown inside-out and wrapping it into a bundle. By the end of this process, only the inside of the gown remains visible.

- Disinfect hands again.

- Remove the goggles/face shield from behind, being careful not to touch the front part.

- Dispose of the equipment and again disinfect hands.

- Remove the respirator by touching the straps only, beginning with the lower ones. Be careful not to touch the outside of the respirator.

- Disinfect hands again.

- Remove gloves and wash and disinfect hands again.

\section{Patient transit to a radiology department; chest X-rays/computed tomography scanning in patients with SARS-CoV-2 infection; post-procedural decontamination of a scanner}

Procedures are developed individually for each $\mathrm{RD}$, with account taken of local conditions (numbers of technicians operating scanners by day or night, availability of nurses at all times, stock of PPE).

\section{Referral/transport/waiting room:}

- The RD is notified in advance of an upcoming procedure [11]. A patient's referral (preferably electronic) should make it clear that COVID-19 infection is suspected or confirmed, and the risk group should be specified, at least with a division into suspected or confirmed infection.

- The patient arrives at the RD with an Emergency Department (ED) team. The patient must be wearing a surgical mask [11].

- The transit route is marked and secured, and other patients should not take this route [7].

- No other patients may be present in the RD waiting room [7].

Use of portable X-ray machines:

- Portable X-ray machines should be used to give chest $\mathrm{X}$-rays (CXR) to patients with (confirmed/suspected) SARS-CoV2 infection $[8,9]$.

- Optimally, these machines should remain where patients with COVID-19 are treated [11].

- The examining radiographer puts on PPE before entering the patient's room.

- Plastic covers on cables are recommended, plastic foil on keyboards and the X-ray unit [7].

- Having performed the examination, the radiographer removes one pair of gloves before disinfecting the machine with disinfecting wipes.

- The radiographer takes the machine out of the room before disinfecting it a second time.

Performing chest X-rays/computed tomography:

The patient is admitted by a radiographer wearing PPE, who is assisted by the ED/department team arriving with the patient.

Computed tomography scanning without contrast:

- Routinely, a CT scan for COVID-19 is performed with no intravenous contrast administered [8].

- CT scan performed by two radiographers (optimal variant): a radiographer wearing PPE and two pairs of

Table 1. Personal protective equipment for staff in contact with patients with suspected or confirmed COVID-19 infection

\begin{tabular}{|c|c|c|}
\hline Optimal equipment & $\begin{array}{l}\text { Acceptable equipment (in the absence } \\
\text { of optimal equipment) }\end{array}$ & $\begin{array}{l}\text { Equipment when contact is with intubated } \\
\text { patients }\end{array}$ \\
\hline $\begin{array}{l}\text { Dedicated waterproof/impermeable gown } \\
\text { Goggles } \\
\text { FFP2/3 respirator } \\
\text { Two pairs of gloves [12] }\end{array}$ & $\begin{array}{l}\text { Nonwoven surgical head cap } \\
\text { Nonwoven gown + plastic surgery gown (on top) } \\
\text { Face shield } \\
\text { FFP2/3 respirator } \\
\text { Two pairs of gloves [12] }\end{array}$ & $\begin{array}{l}\text { Nonwoven surgical head cap } \\
\text { Nonwoven gown } \\
\text { Face shield } \\
\text { Surgical mask } \\
\text { Two pairs of gloves [12] }\end{array}$ \\
\hline
\end{tabular}


gloves positions the patient for examination - having done that, he/she remains in the dirty area and does not enter the control room (does not touch the keyboard). The second radiographer remains in the control room, operates the operating console, and plans and performs the examination.

- CT scan performed by one radiographer (acceptable variant): a radiographer wearing PPE and two pairs of gloves positions the patient for examination, removes one pair of gloves, puts on another (clean) pair, and performs CT examination (preferably in a standing position, not touching anything except the keyboard). The keyboard of the operating console may be wrapped in a transparent plastic bag. After the examination, the radiographer discards this plastic bag and disinfects the keyboard and desk top. In this variant, the control room will also need disinfection following the examination.

- The same principles apply as X-rays are given (by one or two radiographers).

\section{Computed tomography scanning with contrast:}

- Contrast-enhanced CT is performed in certain situations, including where it is suspected that patients with COVID-19 have concomitant diseases/complications (e.g. suspected pulmonary embolism, aortic dissection).

- The patient arrives at the RD with an intravenous (IV) cannula already inserted (from the ED/Department) and is accompanied by a nurse or a paramedic from the ED/ward/emergency service, who connects the contrast-injection system.

Procedure following computed tomography/X-ray examination:

- The X-ray/CT machine is wiped thoroughly with disinfecting wipes [9] - the device is disinfected from the "cleanest" parts (outer parts of the device and where the patient's legs have been resting) to the "dirtiest" (inner parts of the device and where the patient's head has been resting). Desk tops in the room are also wiped.

- The technician (or other staff member in contact with the patient or CT machine) removes PPE carefully, following guidelines and taking precautions [7].

- Reusable components are cleaned, disinfected, and/ or sterilised in accordance with instructions from the manufacturer [11].

- Disposable items are discarded into the special container designated for this and sent for specialist disposal [11].

- If decontamination is available in the hospital, it is performed by a special team following disinfection.

- Depending on local conditions, 30-60 minutes of air exchange is performed following decontamination.

- Following disinfection, (optional) decontamination, and air exchange, the room is ready for other patients.

\section{Indications for imaging studies in patients with suspected or confirmed SARS-CoV-2 infection}

\section{Chest X-ray}

CXR with a portable X-ray machine is recommended as the first and primary imaging study, offering adequate lung assessment in the majority of cases, while making transit of the contagious patient within the hospital unnecessary. It is recommended that the $\mathrm{X}$-ray machine is located on the ward with SARS-CoV-2-infected patients.

If the lung lesions are mild, the CXR may yield falsenegative results.

\section{Indications:}

- Patients with no symptoms or mild symptoms do not require imaging studies.

- While CXR does not provide for COVID-19 diagnosis, it may prove useful as a first examination where SARS-CoV-2 infection is suspected, potentially allowing for an initial diagnosis $[8,9]$.

- Follow-up CXR should be avoided, unless this is required to monitor progress of the disease and will impact upon patient management $[8,16]$.

- In patients with acute respiratory distress syndrome (ARDS), a portable radiograph remains the method of choice, particularly for ventilated patients [17].

\section{Chest computed tomography}

Chest CT may fail to show any abnormalities in the early stages of SARS-CoV-2 infection.

While chest CT proves highly sensitive in detecting lung lesions, its specificity is very low. Similar findings may therefore be made in patients with other types of pneumonia, including that caused by other viruses (H1N1 influenza, adenovirus, CMV) or fungi (pneumocystosis caused by Pneumocystis jiroveci or P. carinii), COP (cryptogenic organising pneumonia), drug-induced pulmonary toxicity, hypersensitivity, and autoimmune disease.

Non-contrast high-resolution computed tomography remains the method of choice in patients with suspected or confirmed COVID-19 [17]. However, the specifics of the protocol applied depend on the CT scanner and the given department.

Contrast-enhanced CT is only indicated where there is a suspicion of coexistent conditions like pulmonary embolism.

\section{Indications:}

- Patients with no symptoms or mild symptoms do not require imaging studies.

- A CT scan should only be ordered in a SARS-CoV2-positive patient if the result will impact upon patient management $[8,16]$. The benefits and risks of the examination should therefore be considered before a CT scan is ordered [8]. 
- In selected cases (e.g. where the waiting time for an RT-PCR test is long, a false negative RT-PCR is suspected, or clinical symptoms suggest COVID-19), a clinician may, along with a radiologist, consider a CT scan - if this will impact upon patient management [8]. While non-specific, a CT image may assist with initial diagnosis of COVID-19 [8], even if final diagnosis requires RT-PCR confirmation $[16,18]$.

- In both stable and unstable disease (Modified Early Warning Score - MEWS $<3$ points or 3-4 points, respectively), CT is highly sensitive at detecting interstitial changes and consolidations, as well as assessing their dynamics $[8,18,19]$. Moreover, along with acidbase balance, it is of predictive value $[19,20]$.

- CXR performed with a portable X-ray machine remains the method of choice in patients in a critical condition, with ARDS, or failure of other organs (MEWS > 4 points) $[16,17]$. In selected cases where CT is required, the patient should be connected to a mobile ventilator to minimise the risk of infection for CT staff [16].

- Chest CT is also indicated in patients with suspected complications, such as empyema or lung abscess, as well as in patients with suspected co-existing conditions like pulmonary embolism.

\section{Chest ultrasound}

Lung ultrasound is not on the training curriculum for radiology residents in Poland and has only been used rarely so far (mainly in children attending highly-specialised centres, with a view to exposure to ionising radiation being minimised). Thus, the majority of radiologists do not have the necessary experience to perform this examination. In addition, there are only a few reports confirming the effectiveness of ultrasound in diagnosing COVID-19.

Pleural ultrasound to visualise effusion should not be confounded with lung ultrasound. However, given the need for only basic experience with ultrasonography, it is easy to diagnose pleural effusion (a symptom seen rarely in COVID-19 patients [21], especially in the early stages of the disease) [22].

Ultrasound is not used to diagnose COVID-19, and it may only confirm the presence of lesions in peripheral parts of lungs. In selected cases, ultrasound can be used to monitor the course of the disease.

\section{Indications:}

- Monitoring peripheral pulmonary lesions in patients with respiratory insufficiency - but only by a team experienced in lung ultrasound.

\section{Course of the examination:}

- The examination extends to the entire available chest area.

- The probe is applied in standardised areas specified in the guidelines for lung and pleural ultrasound.
- In ventilated patients in a critical condition, the examination is most often limited to the anterior and lateral areas.

- In patients in the prone position, the examination involves posterior and lateral areas of the chest.

- It is typical for a convex probe to be used.

- A linear probe may also be deployed, to assess the pleural line and detect consolidations.

- The probe is applied perpendicular to the ribs so at least two adjacent ribs and the pleural line between them are visualised.

\section{Echocardiography}

- Echocardiography is indicated in patients with COVID-19 suffering from suspected acute heart failure in the course of respiratory failure.

\section{Chest magnetic resonance imaging}

- Chest MRI does not play a significant role in COVID-19 diagnosis. Furthermore, as disinfection and decontamination of an MRI machine is difficult, MRI is not indicated in COVID-19 patients.

\section{Conclusions}

1. Imaging studies allow lung lesions to be visualised but do not provide COVID-19 diagnosis, which is based on a positive PCR test result.

2. Chest CT and CXR are of high sensitivity, but very low specificity, when it comes to diagnosing COVID-19.

3. Imaging studies (CXR, CT) should only be performed where results can alter patient management.

4. CXR using a portable unit is recommended, on a point-of-care testing basis.

5. Routinely, it is non-contrast high-resolution chest CT that is performed on COVID-19 patients.

6. Contrast-enhanced chest CT is only performed where there is a suspicion of comorbid conditions, such as pulmonary embolism.

7. Clear information regarding suspected/confirmed COVID-19 is required on the referral, because better protection of RD staff and patients is then possible.

8. Staff of an RD examining a patient with suspected or confirmed COVID-19 infection must be provided with adequate PPE (in line with PMSR guidelines).

9. Lung ultrasound is a specialised examination not suitable for diagnosing COVID-19. This examination is only recommended for physicians with extensive experience in this field.

10. MRI is not recommended for COVID-19 patients.

\section{Conflict of interest}

The authors report no conflict of interest. 


\section{References}

1. Huang C, Wang Y, Li X, et al. Clinical features of patients infected with 2019 novel coronavirus in Wuhan, China. Lancet 2020; 395: 497-506.

2. Sun P, Lu X, Xu C, et al. Understanding of COVID-19 based on current evidence. J Med Virol 2020. doi: 10.1002/jmv.25722 [In press]

3. Coronavirus disease 2019 (COVID-19): situation report, 51 [editorial]. 2020 [cited 2020 Mar 27]; 1: [9 screens]. Available at: https:// www.who.int/docs/default-source/coronaviruse/situation-reports/20200311-sitrep-51-covid-19.pdf?sfvrsn=1ba62e57_10.

4. Zu ZY, Jiang MD, Xu PP, et al. Coronavirus disease 2019 (COVID-19): a perspective from China. Radiology 2020. doi: 10.1148/radiol. 2020200490 [In press].

5. COVID-19: BSTI Statement and Guidelines [editorial]. 2020 [cited 2020 Mar 27]; 1: [28 screens]. Available at: URL: https://www.bsti. org.uk/media/resources/files/BSTI_COVID-19_Radiology_Guidance_version_2_16.03.20.pdf.

6. Kanne JP, Little BP, Chung JH, et al. Essentials for radiologists on COVID-19: an update - radiology scientific expert panel. Radiology 2020. doi: 10.1148/radiol.2020200527 [In press].

7. Huang Z, Zhao S, Li Z, et al. The battle against coronavirus disease 2019 (COVID-19): emergency management and infection control in a radiology department. J Am Coll Radiol 2020. doi: 10.1016/j. jacr.2020.03.011 [In press].

8. Mossa-Basha M, Meltzer CC, Kim DC, et al. Radiology department preparedness for COVID-19: radiology scientific expert panel. Radiology 2020. Available at: https://pubs.rsna.org/doi/10.1148/ radiol.2020200988.

9. Kooraki S, Hosseiny M, Myers L, et al. Coronavirus (COVID-19) outbreak: what the department of radiology should know. J Am Coll Radiol 2020; 17: 447-451.

10. Kampf G, Todt D, Pfaender S, et al. Persistence of coronaviruses on inanimate surfaces and their inactivation with biocidal agents. J Hosp Infect 2020; 104: 246-251.

11. World Health Organization. Infection prevention and control during health care when COVID-19 is suspected. Interim guidance [editorial]. 2020 [cited 2020 Mar 27]; 1: [5 screens]. Available at: https://apps.who.int/iris/bitstream/handle/10665/331495/WHO2019-nCoV-IPC-2020.3-eng.pdf.
12. Cheung JCH, Ho LT, Cheng JV, et al. Staff safety during emergency airway management for COVID-19 in Hong Kong. Lancet Respir Med 2020; 8: e19.

13. Rymer W, Wroczyńska A, Matkowska-Kocjan A. COVID-19-aktualny stan wiedzy. Med Prakt 2020; 3: 102-121.

14. Lee SA, Grinshpun SA, Reponen T. Respiratory performance offered by $\mathrm{N} 95$ respirators and surgical masks: human subject evaluation with $\mathrm{NaCl}$ aerosol representing bacterial and viral particle size range. Ann Occup Hyg 2008; 52: 177-185.

15. Mitchell R, Roth V, Gravel D, et al. Are health care workers protected? An observational study of selection and removal of personal protective equipment in Canadian acute care hospitals. Am J Infect Control 2013; 41: 240-244.

16. ACR Recommendations for the use of Chest Radiography and Computed Tomography (CT) for Suspected COVID-19 Infection [editorial]. 2020 [cited 2020 Mar 27]; 1: [6 screens]. Available at: https:// www.acr.org/Advocacy-and-Economics/ACR-Position-Statements/ Recommendations-for-Chest-Radiography-and-CT-for-SuspectedCOVID19-Infection.

17. Jin YH, Cai L, Cheng ZS, et al. A rapid advice guideline for the diagnosis and treatment of 2019 novel coronavirus (2019-nCoV) infected pneumonia (standard version). Mil Med Res 2020; 7: 4.

18. Fang Y, Zhang H, Xie J, et al. Sensitivity of chest CT for COVID-19: comparison to RT-PCR. Radiology 2020. doi: 10.1148/radiol. 2020200432 [In press].

19. Song F, Shi N, Shan F, et al. Emerging 2019 novel coronavirus (2019nCoV) pneumonia. Radiology 2020; 295: 210-217.

20. Salehi S, Abedi A, Balakrishnan S, et al. Coronavirus disease 2019 (COVID-19): a systematic review of imaging findings in 919 patients. AJR Am J Roentgenol 2020. doi: 10.2214/AJR.20.23034 [In press].

21. Shi H, Han X, Jiang N, et al. Radiological findings from 81 patients with COVID-19 pneumonia in Wuhan, China: a descriptive study. Lancet Infect Dis 2020; 20: 425-434.

22. Buonsenso D, Pata D, Chiaretti A. COVID-19 outbreak: less stethoscope, more ultrasound. Lancet Respir Med 2020. doi: 10.1016/ S2213-2600(20)30120-X [In press]. 